\title{
Palhaçoterapia em ambiente hospitalar: uma revisão de literatura
}

\author{
Clown therapy in the hospital setting: A review of the literature
}

Soraia de Camargo Catapan (http://orcid.org/0000-0001-6223-1697) ${ }^{1}$

Walter Ferreira de Oliveira (http://orcid.org/0000-0002-1808-0681) ${ }^{1}$

Tatiana Marcela Rotta (https://orcid.org/0000-0002-9125-0819) ${ }^{1}$

${ }^{1}$ Programa de PósGraduação em Saúde Coletiva, Universidade Federal de Santa Catarina. R. Delfino Conti Bloco H, Trindade. 88040-900 Florianópolis SC Brasil. scatapan@gmail.com

\begin{abstract}
The presence of clowns in hospitals is relatively recent and more than 700 organizations now perform clown therapy in hospitals in Brazil and around the world. Considering the emphasis on comprehensive care and the humanization of health, it is timely to understand and analyze the national and international scientific literature on clown therapy. This practice challenges the hegemonic biomedical model and potentially helps in the recuperation of health. A review of the literature was conducted and 18 publications were analyzed using thematic content analysis. The following categories arose from the analysis: physiological, behavioral, and emotional responses; presurgical anxiety and attribution of new meanings to practices and the hospital environment. Results have shown that clown therapy leads to a significant decrease in preoperative anxiety in children, even compared with the results obtained with the use of anesthesia-inducing drugs. Most studies investigated the use of clown therapy during patients' adaptation to hospitalization, helping them to attribute new meanings to this situation. Despite its low representation in the scientific field and the fact that it has not been widely accepted as a health practice, several studies have shown the benefits of using clown therapy in the hospital setting.
\end{abstract}

Key words Humanization of care, Laughter therapy, Hospital care
Resumo A inserção do palhaço no hospital é relativamente recente e mais de 700 organizações realizam a palhaçoterapia nessas instituições no Brasil e em outras ao redor do mundo. Dada a ênfase na integralidade do cuidado e humanização da assistência à saúde, torna-se oportuno compreender e analisar a produção nacional e internacional do conhecimento científico sobre esta prática que desafia o modelo hegemônico de cuidado e potencialmente contribui na recuperação da saúde. Foi realizada uma revisão de literatura utilizando análise de conteúdo temática que analisou 18 publicações. As categorias que emergiram foram: respostas fisiológicas, comportamentais e emocionais; ansiedade pré-operatória; e ressignificação das práticas e do ambiente hospitalar. A literatura analisada aponta que a palhaçoterapia provoca diminuição significativa da ansiedade pré-operatória em crianças, comparativamente maior do que a obtida com o uso de um fármaco indutor de anestesia. A maior parte dos estudos investigou o uso da palhaçoterapia na adaptação à hospitalização, auxiliando na atribuição de novos significados. Apesar da baixa representatividade do tema no campo científico e mesmo não sendo amplamente aceita como prática de saúde, os resultados encontrados sugerem benefícios na utilização da palhaçoterapia em ambiente hospitalar.

Palavras-chave Humanização da assistência, Terapia do riso, Assistência hospitalar 


\section{Introdução}

Diversas ações de humanização da assistência hospitalar têm sido adotadas mundialmente. Mais do que humanizar o atendimento, visam melhorar as relações entre profissionais da saúde e pacientes e junto às instituições de saúde. Essas ações buscam resgatar valores como solidariedade, colaboração, afetividade nas relações, respeito à diversidade, valorização das queixas, cuidado com o outro, em contraponto a lógica atual que privilegia o lucro, as formas de exclusão, o mercado e a competitividade, entre outros valores "desumanizantes".

A atuação de palhaços em hospitais visa integrar um cuidar eficiente e um cuidar mais humano, em consonância com o conceito ampliado de saúde, considerando o ser humano todo em suas multiplicidades, para além do corpo físico. A centralidade deixa de ser a doença, o doente ou seus sintomas físicos e passa a ser a pessoa, sua nova realidade institucionalizada e os sentimentos consequentes dessas alterações.

A palhaçoterapia tem diversas definições, nomenclaturas, atores, finalidades, abordagens e públicos. Uma de suas definições, feita por Dionigi et al. ${ }^{2}$, assume tratar-se da "implementação de técnicas de palhaço derivadas da arte circense, para o contexto da doença, no intuito de melhorar o humor das pessoas e seu estado mental" (tradução dos autores). Seu foco são as necessidades subjetivas dos pacientes, as quais direcionam a dinâmica e o improviso das interações realizadas pelos palhaços, em sua maioria não expressas nas queixas ou prontuários médicos.

Dionigi et al. ${ }^{2}$ ressaltam ainda que os palhaços provavelmente existem desde Hipócrates, já que os médicos daquela época acreditavam que o bom humor influenciava positivamente o processo de cura. Entretanto a valorização do uso do humor na medicina é recente e deriva principalmente do questionamento do modelo biomédico hegemônico ${ }^{3}$. O primeiro relato da presença de palhaços em hospitais foi em Londres, em uma enfermaria infantil, no ano de $1908^{3}$. Mas sua efetiva inserção cita nomes contemporâneos, como Patch Adams, do Gesundheit Institute, idealizado no início da década de $1970^{4}$ e Michael Christensen, do Big Apple Circus Clown Care Unit, fundado em $1986^{5}$.

A prática da palhaçoterapia no Brasil iniciouse em 1991, trazida de Nova Iorque por Wellington Nogueira que trabalhou na Clown Care Unit e aqui fundou os Doutores da Alegria, organização da sociedade civil que influenciou muitos outros grupos, tanto na prática quanto na pesquisa da palhaçoterapia ${ }^{5}$. Um levantamento feito em 2012, indicou que mais de 700 grupos de palhaços profissionais e amadores realizam ações e formações de palhaços de hospitais no Brasil ${ }^{6}$ e tantos outros ao redor do mundo. Além dos benefícios terapêuticos dessa prática aos pacientes internados, muitos projetos também visam uma formação mais humanística de seus integrantes, normalmente estudantes universitários de diversas áreas ${ }^{6}$.

Dada a ênfase em integralidade do cuidado e humanização da assistência, no âmbito da saúde brasileira, torna-se oportuno compreender e analisar o conhecimento científico produzido nacional e internacionalmente sobre a palhaçoterapia em ambiente hospitalar. Portanto, esse é um estudo de revisão que buscou nas principais bases de dados trabalhos referentes à atuação do palhaço no contexto hospitalar, considerando suas utilizações e resultados, junto a pacientes adultos e crianças.

\section{Método}

Foi realizada uma revisão de literatura com base nas diretrizes do Preferred Reporting Items for Systematic reviews and Meta-Analyses (PRIS$\mathrm{MA})^{7}$, cujos conceitos e tópicos gerais são importantes não só para revisões sistemáticas, mas também para outros estudos de revisão. O Prisma Statement, composto por um fluxograma e um checklist, ordena as etapas de identificação, rastreamento, seleção e análise de publicações, evitando direcionamento dos resultados?.

As bases de dados utilizadas incluíram a Biblioteca Virtual de Saúde (BVS), englobando periódicos das bases de dados do Sistema Latino Americano e do Caribe de Informação em Ciências da Saúde (Lilacs), a Base de Dados em Enfermagem (BDENF), o Index Psicologia (IndexPsi) e o Îndice Bibliográfico Espanhol de Ciências da Saúde (IBECS). Também foram utilizadas as bases Scientific Electronic Library Online (SciELO), Scopus e Publisher Medline (PubMed).

As buscas foram realizadas em maio de 2016, sem restrição de período inicial ou tipo de documento, nos idiomas inglês, português e espanhol. Foram empregados descritores e/ou palavras chave, de acordo com a base de dados, suas derivações e traduções em inglês e espanhol, assim como combinações utilizando operadores boleanos. As chaves de busca utilizadas em cada base de dados e o número total de publicações encontradas estão descritas no Quadro 1. 
Quadro 1. Chaves de busca por bases de dados e número de publicações encontradas.

\begin{tabular}{|c|c|c|}
\hline Bases de Dados & Chaves de Busca & No \\
\hline $\begin{array}{l}\text { Lilacs, IBECS, } \\
\text { INDEXPSI e } \\
\text { BDENF, via BVS }\end{array}$ & 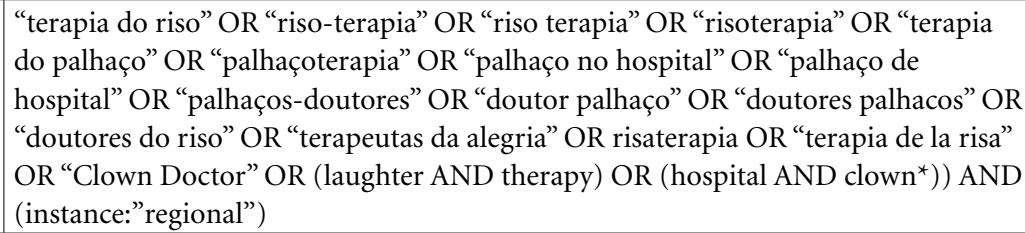 & 70 \\
\hline $\begin{array}{l}\text { SciELO } \\
\text { Regional }\end{array}$ & 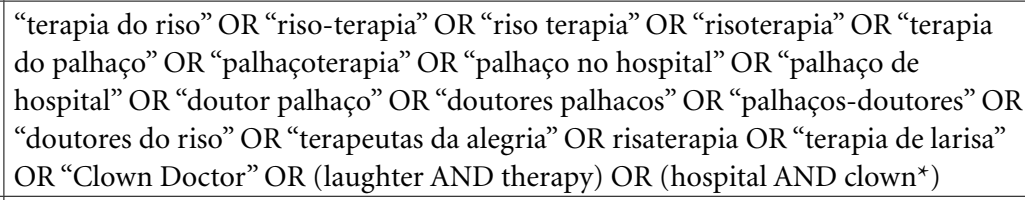 & 17 \\
\hline $\begin{array}{l}\text { PubMed / } \\
\text { Medline }\end{array}$ & $\begin{array}{l}\text { "laughter therapy"[MeSH Terms] OR ("laughter"[All Fields] AND “therapy"[All } \\
\text { Fields]) OR “Clown Doctor”[All Fields] OR (("hospitals"[MeSH Terms] OR } \\
\text { "hospitals"[All Fields] OR "hospital”[All Fields]) AND ("clown"[All Fields] } \\
\text { OR “clowns"[All Fields] OR "clowning"[All Fields])) AND (English[lang] OR } \\
\text { Portuguese[lang] OR Spanish[lang]) }\end{array}$ & 423 \\
\hline SCOPUS & 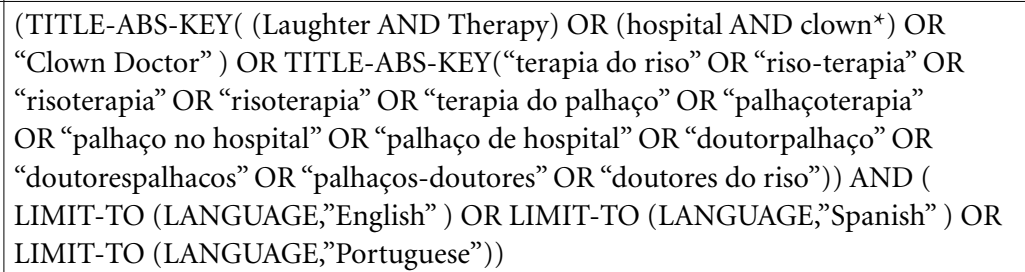 & 443 \\
\hline
\end{tabular}

Os critérios de exclusão adotados para seleção dos artigos para análise, considerando o objetivo desse estudo, foram: a) quanto ao local, estudos sobre a utilização da palhaçoterapia em ambientes diferentes do hospitalar; b) referentes aos participantes da pesquisa, intervenções com foco nas alterações provocadas somente nos pais ou acompanhantes, profissionais de saúde ou palhaços; c) quanto ao método utilizado, estudos que apresentavam resultados sem menção do método de análise de dados e estudos denominados qualitativos, que citavam métodos de coleta e análise quantitativos apenas; d) referentes aos tipos de estudo, relatos de experiência; e) pesquisas sobre intervenções esporádicas ou com profissionais de saúde ou estudantes treinados apenas para fins do estudo.

Referente ao último critério de exclusão citado (e), apesar da inexistência de um número expressivo de estudos sobre a palhaçoterapia, acredita-se que para que uma pesquisa sobre este tema tenha resultados confiáveis, sua prática deve ter alguns requisitos mínimos, dada sua heterogeneidade. Entre esses requisitos, ressaltamos a preparação para exercer a função de palhaço no ambiente hospitalar. Não se trata de formação como palhaço profissional, mas a aquisição de habilidades artísticas e a preparação para exerce-las nesse ambiente. Entende-se também que a continuidade do trabalho é parte fundamental da ação terapêutica.

Para análise das publicações selecionadas foi utilizada a análise de conteúdo temática, adaptando os passos sugeridos por Minayo ${ }^{8}$, na seguinte sequência: leitura inicial do material selecionado; leitura compreensiva buscando apreender semelhanças e particularidades do conjunto; agrupamento em temas de acordo com os núcleos de sentido identificados; e síntese interpretativa do conjunto de publicações agrupadas, fazendo emergir categorias.

\section{Resultados e discussão}

A busca descrita encontrou um total de 953 publicações. O software Endnote Web foi utilizado para gerenciar as referências bibliográficas ${ }^{9}$. Após remoção de 235 títulos por duplicidade, a amostra ficou reduzida a 718. Na filtragem manual pelo título foram removidas 533 publicações que não tinham relação com o tema de estudo, principalmente pela existência de um tipo de tumor nasal chamado clown nose ${ }^{10}$. Posteriormente, com o 
rastreamento por título e resumo, foram removidas outras 129 publicações que não tinham relação com o tema, não se encontravam disponíveis ou, apesar de terem o título em inglês, português ou espanhol, foram publicados em outro idioma. Às 56 publicações restantes foram adicionadas posteriormente outras 16 encontradas por busca manual, com base em referências bibliográficas do material encontrado.

No total, 72 publicações foram lidas integralmente e descartadas 54 de acordo com os critérios de exclusão já expostos. Assim, restaram para análise 18 publicações. A Figura 1 apresenta o fluxograma da revisão de literatura, conforme as etapas preconizadas por Moher et al. ${ }^{7}$.

A leitura inicial das publicações selecionadas permitiu a listagem de alguns pontos principais apresentados no Quadro 2: autores principais, ano de publicação, título do trabalho e breve resumo contendo objetivos, métodos e principais resultados encontrados.

Quanto a terminologia adotada neste trabalho, faz-se necessária uma breve explanação, já que esta não é uniforme, conforme demonstra o Quadro 2. Masetti1 ${ }^{11}$ justifica a adoção do termo clown (palhaço, em inglês) no Brasil pela diferença entre palhaços de circo e os que atuam em outros espaços cênicos para plateias específicas, como é o caso dos palhaços de hospital. Preferimos adotar esse último termo neste estudo. Diversas nomenclaturas foram encontradas para as terapias que utilizam palhaços treinados especificamente para atuar em hospitais, tais como therapeutic clown ${ }^{12}$, clown therapy ${ }^{13}$, clown care $^{3}$ ou uma mistura de idiomas como clownterapia ${ }^{14}$ e arte $c l o w^{10}$. Para este trabalho, adotamos o termo palhaçoterapia, ressaltando o potencial terapêutico da interação entre palhaço e paciente, no idioma de publicação deste trabalho.

A partir da visão geral indicada no Quadro 2 , pode-se partir para a análise dos resultados. Dos 18 trabalhos analisados, 15 foram publicados no período compreendido entre 2009 a 2016, evidenciando a contemporaneidade do tema, conforme pode-se visualizar no Gráfico 1. Este gráfico denota ascendência no número de publicações de 2012 a 2014, com posterior queda, já que nenhum trabalho selecionado foi publicado em 2015 e posterior aumento com dois trabalhos publicados em 2016.

Apesar da presença de palhaços em hospitais ter sido relatada pela primeira vez em Londres no início do século passado, sua efetiva inserção ocorreu nas décadas de 70 e 80 , com destaque para os trabalhos de Patch Adams ${ }^{4}$ e Michael
Christensen ${ }^{3}$. Esta inserção relativamente recente no contexto hospitalar pode elucidar a contemporaneidade das publicações sobre o tema. Por outro lado, a proposta de uma prática inovadora que se contrapõe à lógica do modelo biomédico hegemônico pode ser um motivo que corrobora para o crescente interesse pelo tema no campo científico.

O Quadro 3 fornece uma visão geral das semelhanças e particularidades do conjunto obtidas após a leitura compreensiva. A partir da sua construção foi possível a identificação de núcleos de sentido, agrupamento em temas e consequente emersão de categorias de análise, para melhor orientar a discussão. Esse processo foi orientado pelo enfoque principal dos trabalhos, considerando as utilizações e resultados da palhaçoterapia junto a pacientes hospitalares.

Indicado no Quadro 3, quanto ao país de origem, Itália, Brasil, Suécia e Estados Unidos foram os países com maior número de publicações e o restante dividiu-se igualmente entre Portugal, Austrália, Canadá e Finlândia.

Dentre as 18 publicações analisadas encontrase um capítulo de livro, dois artigos com análise de dados secundários, sendo uma revisão de literatura e uma meta análise. As outras 15 pesquisas incluíram a coleta de dados primários, sendo 14 artigos e uma tese e, destas, nove tinham como população do estudo apenas crianças e seis, além de crianças, seus pais ou responsáveis. Nota-se a ausência de estudos com foco nos efeitos e utilizações da palhaçoterapia em pacientes adultos hospitalizados apesar da existência de grupos de palhaços que realizam visitas a pacientes adultos em hospitais do Brasil e do mundo.

Quanto ao método, nove trabalhos utilizaram metodologia quantitativa, sendo sete ensaios clínicos com grupos de controle. Estudos qualitativos somaram-se oito, sendo dois estudos fenomenológicos, uma revisão de literatura de dados qualitativos, uma meta análise, um estudo qualitativo com técnicas da teoria fundamentada nos dados ${ }^{15}$ e três estudos utilizaram análise de conteúdo para tratamento dos dados. Observa-se que os estudos quantitativos analisados seguem um desenho semelhante, enquanto os estudos qualitativos são bastante heterogêneos, adotando abordagens diversas, dada sua natureza exploratória aplicada a um fenômeno em ascensão no campo científico.

Ainda no Quadro 3, a utilização do método de análise de conteúdo temática ${ }^{8}$ para as 18 publicações encontradas propiciou que emergissem três categorias. A categoria menos expressiva agrupou três publicações com o objetivo comum de quantificar os efeitos da palhaçoterapia ava- 


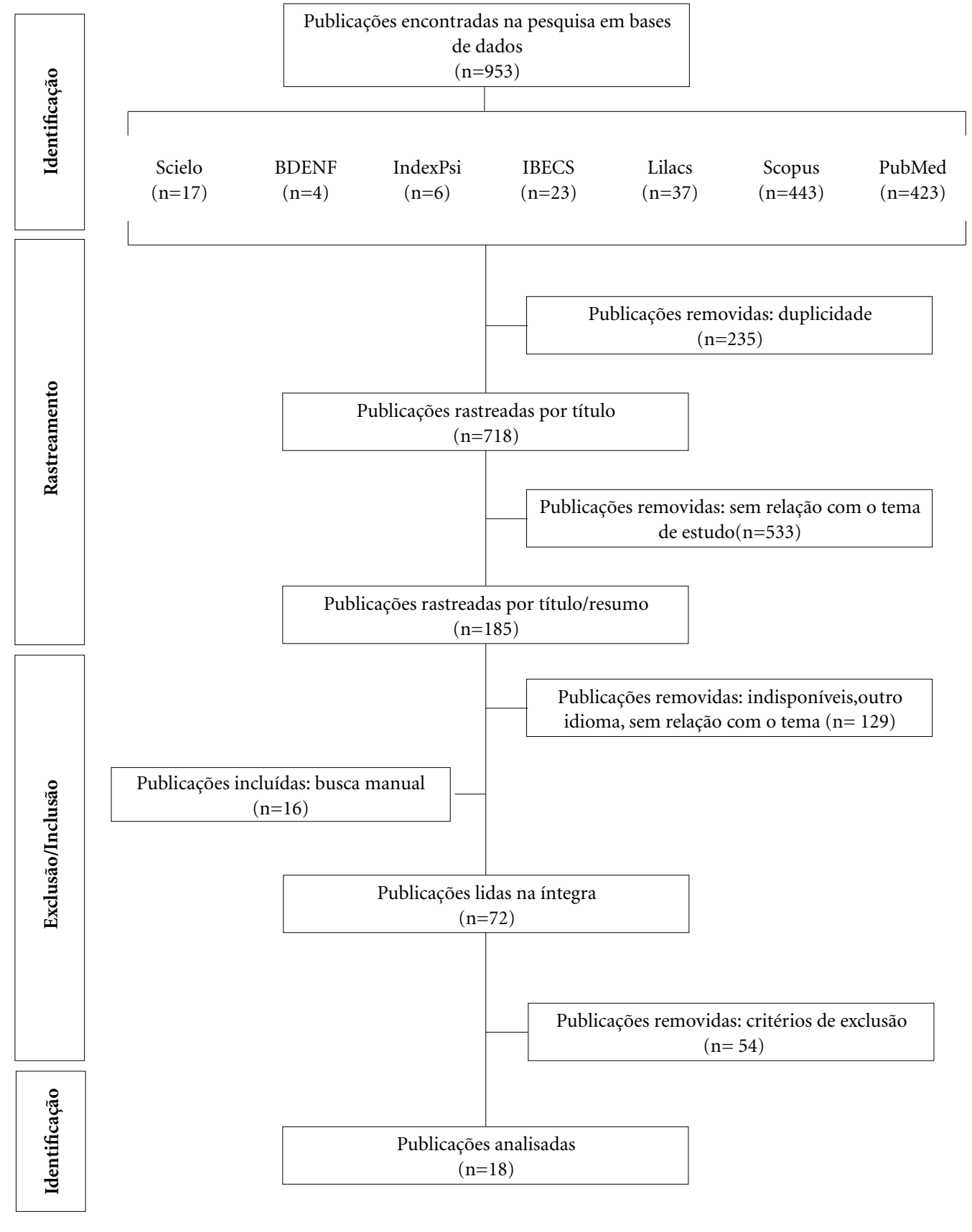

Figura 1. Fluxograma da revisão de literatura adaptado de Moher et al .

liando respostas fisiológicas, comportamentais e emocionais, utilizando escalas e variáveis individuais. Optou-se por denominar esta categoria 'Respostas fisiológicas, comportamentais e emocionais'. Uma segunda categoria referindo-se aos efeitos da palhaçoterapia sobre a ansiedade pré-operatória agrupou seis artigos e manteve esta mesma denominação. A categoria mais expressiva, que agrupou nove publicações, diz respeito as influências sobre as práticas hospitalares e sobre os significados atribuídos pelos pacientes à sua estada no hospital, categoria denominada 'Ressignificação das práticas e do ambiente hospitalar'. 
Quadro 2. Resumo das publicações selecionadas para análise.

\begin{tabular}{|c|c|c|}
\hline Autores e data & Título & Resumo \\
\hline $\begin{array}{l}\text { Aquino RG et } \\
\text { al., } 2004^{16}\end{array}$ & $\begin{array}{l}\text { Doutores da graça: a } \\
\text { criança fala. }\end{array}$ & $\begin{array}{l}\text { Identificou a opinião de crianças hospitalizadas sobre os Doutores } \\
\text { da Graça utilizando análise de conteúdo. Concluiu que o palhaço } \\
\text { tem o poder de diminuir as dores, deixando-as mais fortes, } \\
\text { autônomas, bem-humoradas e alegres, suprindo a necessidade de } \\
\text { brincar, compartilhando olhares e emoções. }\end{array}$ \\
\hline $\begin{array}{l}\text { Bertini M et al., } \\
2011^{17}\end{array}$ & $\begin{array}{l}\text { Clowns benefit children } \\
\text { hospitalized for } \\
\text { respiratory pathologies. }\end{array}$ & $\begin{array}{l}\text { Investigou os efeitos da presença de um palhaço na evolução } \\
\text { clínica de crianças com patologias respiratórias utilizando } \\
\text { grupo controle. As crianças do grupo experimental mostraram } \\
\text { desaparecimento dos sintomas, diminuição de pressão arterial, } \\
\text { frequência respiratória, temperatura e dor em comparação ao } \\
\text { grupo controle. Concluiu-se que a palhaçoterapia pode melhorar } \\
\text { a recuperação clínica das crianças. }\end{array}$ \\
\hline $\begin{array}{l}\text { Dionigi A et al., } \\
2012^{2}\end{array}$ & Clowns in hospitals. & $\begin{array}{l}\text { Capitulo do livro 'Humor and Health Promotion' contextualizou } \\
\text { a palhaçoterapia, seu desenvolvimento e utilização. Ressaltou } \\
\text { a importância de esclarecer o papel do palhaço devido sua } \\
\text { desvalorização e a necessidade de proporcionar formação } \\
\text { adequada, pois eles têm como missão um desafio: trabalhar com } \\
\text { as emoções dos pacientes. }\end{array}$ \\
\hline $\begin{array}{l}\text { Dionigi A et al., } \\
2014^{18}\end{array}$ & $\begin{array}{l}\text { Clown intervention to } \\
\text { reduce preoperative } \\
\text { anxiety in children and } \\
\text { parents: a randomized } \\
\text { controlled trial. }\end{array}$ & $\begin{array}{l}\text { Investigou se a intervenção do palhaço poderia reduzir a } \\
\text { ansiedade pré-operatória em crianças, utilizando grupo controle } \\
\text { randomizado. A intervenção clown reduziu a ansiedade das } \\
\text { crianças que apresentaram melhor adaptação do que as do grupo } \\
\text { controle. O mesmo aconteceu com os pais. }\end{array}$ \\
\hline $\begin{array}{l}\text { Esteves CH et } \\
\text { al., 2014 }\end{array}$ & $\begin{array}{l}\text { Humanização em } \\
\text { contexto pediátrico: o } \\
\text { papel dos palhaços na } \\
\text { melhoria do ambiente } \\
\text { vivido pela criança } \\
\text { hospitalizada. }\end{array}$ & $\begin{array}{c}\text { Revisão de literatura que refletiu sobre a humanização de espaços, } \\
\text { rotinas e atmosferas, e os atenuantes das experiências vividas na } \\
\text { internação, incluindo a intervenção dos palhaços de hospital. A } \\
\text { intervenção dos palhaços promove expressão livre da criança, } \\
\text { de sua autonomia, criatividade, exploração e conhecimento do } \\
\text { mundo e desenvolvimento psicossocial. }\end{array}$ \\
\hline $\begin{array}{l}\text { Fernandes SC, } \\
\text { Arriaga P, 2010 }\end{array}$ & $\begin{array}{l}\text { The effects of clown } \\
\text { intervention on worries } \\
\text { and emotional responses } \\
\text { in children undergoing } \\
\text { surgery. }\end{array}$ & $\begin{array}{c}\text { Estudou o efeito da intervenção do palhaço na redução da } \\
\text { ansiedade pré-operatória e das respostas emocionais em crianças } \\
\text { utilizando grupo controle. Houve redução na ansiedade não só } \\
\text { nas crianças, mas também em seus pais. As crianças sentiram-se } \\
\text { mais calmas e felizes. A opinião dos profissionais de saúde sobre a } \\
\text { intervenção foi favorável. }\end{array}$ \\
\hline $\begin{array}{l}\text { Ford K et al., } \\
2013^{15}\end{array}$ & $\begin{array}{l}\text { More than just clowns } \\
\text { - Clown doctor rounds } \\
\text { and their impact for } \\
\text { children, families and } \\
\text { staff. }\end{array}$ & $\begin{array}{l}\text { Estudou o impacto da palhaçoterapia em cenário pediátrico } \\
\text { agudo sobre as crianças, famílias, profissionais de saúde e } \\
\text { palhaços, utilizando análise de conteúdo. Rica descrição e } \\
\text { evidências de que o impacto das visitas é vivenciado além da } \\
\text { interação, não articulado anteriormente. }\end{array}$ \\
\hline $\begin{array}{l}\text { Golan G et al., } \\
2009^{21}\end{array}$ & $\begin{array}{l}\text { Clowns for the } \\
\text { prevention of } \\
\text { preoperative anxiety in } \\
\text { children: a randomized } \\
\text { controlled trial }\end{array}$ & $\begin{array}{l}\text { Comparou os efeitos da interação com palhaços na ansiedade } \\
\text { pré-operatória aos efeitos da utilização de midazolam ou } \\
\text { ausência de terapia farmacológica, utilizando grupo controle } \\
\text { randomizado. Concluiu-se que a utilização de palhaços pode } \\
\text { aliviar significativamente a ansiedade pré-operatória antes da } \\
\text { introdução da anestesia. }\end{array}$ \\
\hline $\begin{array}{l}\text { Kingsnorth S et } \\
\text { al., } 2011^{12}\end{array}$ & $\begin{array}{l}\text { Physiological and } \\
\text { emotional responses } \\
\text { of disabled children to } \\
\text { therapeutic clowns: a } \\
\text { pilot study }\end{array}$ & $\begin{array}{l}\text { Estudou os efeitos da intervenção clowning em pacientes } \\
\text { internados em um hospital pediátrico, observando as crianças } \\
\text { em } 4 \text { dias alternando um programa de televisão e a visita } \\
\text { do palhaço. As diferenças mais significativas na intervenção } \\
\text { clowning: alterações no SNA; relatórios de humor, expressões } \\
\text { faciais, emoções e vocalizações mais positivas. Concluíram que o } \\
\text { clowning tem impacto direto e positivo sobre as crianças. }\end{array}$ \\
\hline
\end{tabular}


Quadro 2. Resumo das publicações selecionadas para análise.

\begin{tabular}{|c|c|c|}
\hline Autores e data & Título & Resumo \\
\hline Linge $\mathrm{L}, 2012^{22}$ & $\begin{array}{l}\text { Magical attachment: } \\
\text { Children in magical } \\
\text { relations with hospital } \\
\text { clowns. }\end{array}$ & $\begin{array}{l}\text { Propôs uma compreensão das relações com palhaços de hospital } \\
\text { na perspectiva das crianças, usando teoria psicológica e análise } \\
\text { fenomenológica interpretativa. Caracterizou esse encontro como } \\
\text { uma área mágica e segura, intermediária entre fantasia e realidade. } \\
\text { A ligação entre palhaço de hospital e a criança é temporária, } \\
\text { anônima, reverte papéis e cria uma experiência emocional que } \\
\text { oportuniza transpor barreiras. }\end{array}$ \\
\hline Linge L, $2013^{23}$ & $\begin{array}{l}\text { Joyful and serious } \\
\text { intentions in the work } \\
\text { of hospital clowns: a } \\
\text { meta-analysis based on } \\
\text { a 7-year research project } \\
\text { conducted in three parts. } \\
\end{array}$ & $\begin{array}{l}\text { Meta-análise que propôs entender profundamente os encontros } \\
\text { entre os palhaços de hospitais e crianças. Concluiu que esse } \\
\text { cuidado transcende barreiras, é mágico e seguro. A alegria não } \\
\text { exige algo em troca: um contrapeso positivo ao que era visto como } \\
\text { falta de cuidado médico. Os palhaços usam diferentes soluções } \\
\text { facilitando as coisas para as crianças, pais e funcionários. }\end{array}$ \\
\hline $\begin{array}{l}\text { Mansson ME et } \\
\text { al., } 2013^{24}\end{array}$ & $\begin{array}{l}\text { Use of clowns to aid } \\
\text { recovery in hospitalized } \\
\text { children }\end{array}$ & $\begin{array}{l}\text { Examinou como crianças internadas percebem seus encontros } \\
\text { com os palhaços, utilizando análise do conteúdo. As crianças } \\
\text { experimentaram a internação como divertida, auxiliando a sentir- } \\
\text { se mais em casa. Trouxe brincadeiras e humor, mudando o foco da } \\
\text { atenção, ajudando-as no bem-estar e recuperação. }\end{array}$ \\
\hline $\begin{array}{l}\text { Martins AKL et } \\
\text { al., } 2016^{14}\end{array}$ & $\begin{array}{l}\text { Repercussões } \\
\text { da clownterapia } \\
\text { no processo de } \\
\text { hospitalização da criança }\end{array}$ & $\begin{array}{l}\text { Analisou as repercussões da clownterapia na hospitalização da } \\
\text { criança, utilizando análise de conteúdo. Percebeu a formação } \\
\text { espontânea de vínculos entre equipe de enfermagem, crianças } \\
\text { e responsáveis, facilitando a compreensão e colaboração } \\
\text { no cuidado. A clownterapia é facilitadora das práticas de } \\
\text { enfermagem, trazendo a perspectiva da promoção da saúde. }\end{array}$ \\
\hline $\begin{array}{l}\text { Messina M et } \\
\text { al., } 2014^{13}\end{array}$ & $\begin{array}{l}\text { Preoperative distraction } \\
\text { in children: hand-held } \\
\text { videogames vs clown } \\
\text { therapy }\end{array}$ & $\begin{array}{l}\text { Avaliou a eficácia de terapia do palhaço em reduzir a ansiedade } \\
\text { pré-operatória em crianças, utilizando grupo controle } \\
\text { randomizado. Existe relação positiva entre níveis de ansiedade } \\
\text { na criança e a palhaçoterapia, especialmente durante a indução } \\
\text { da anestesia. A ação do palhaço pode melhorar os cuidados } \\
\text { hospitalares, apesar da resistência da equipe médica na inclusão } \\
\text { desta prática na sala de operação. }\end{array}$ \\
\hline $\begin{array}{l}\text { Saliba FG et al., } \\
2016^{25}\end{array}$ & $\begin{array}{l}\text { Salivary Cortisol Levels: } \\
\text { The Importance of } \\
\text { Clown Doctors to } \\
\text { Reduce Stress. }\end{array}$ & $\begin{array}{l}\text { Correlacionou o entretenimento das atividades de palhaços } \\
\text { em crianças hospitalizadas usando os níveis de cortisol, em } \\
\text { abordagem quantitativa. Cada criança foi o próprio controle em } \\
\text { dois grupos: almoço e jantar. A intervenção dos palhaços é eficaz } \\
\text { na diminuição desse biomarcador do estresse, atenuando os } \\
\text { efeitos da hospitalização, contribuindo para a recuperação. }\end{array}$ \\
\hline $\begin{array}{l}\text { Tan Jr. AK, } \\
2014^{3}\end{array}$ & $\begin{array}{l}\text { A Qualitative } \\
\text { Phenomenographical } \\
\text { Study of the Experience } \\
\text { of Parents with Children } \\
\text { in Clown } \\
\text { Care Services }\end{array}$ & $\begin{array}{l}\text { Descreveu as impressões, experiências, benefícios, barreiras } \\
\text { e impactos dos cuidados de um programa clown sobre pais e } \\
\text { filhos, em estudo fenomenológico. A palhaçoterapia cria um } \\
\text { estado emocional positivo, promove interação e condições para } \\
\text { um ambiental favorável. Os resultados sugerem que a integração } \\
\text { desses cuidados é importante na promoção do bem-estar } \\
\text { emocional e psicossocial. }\end{array}$ \\
\hline $\begin{array}{l}\text { Vagnoli L et al., } \\
2005^{26}\end{array}$ & $\begin{array}{l}\text { Clown Doctors } \\
\text { as a Treatment } \\
\text { for Preoperative } \\
\text { Anxiety in Children: } \\
\text { A Randomized, } \\
\text { Prospective Study } \\
\end{array}$ & $\begin{array}{l}\text { Investigou os efeitos da presença de palhaços na ansiedade } \\
\text { pré-operatória de crianças e pais ou acompanhantes, durante a } \\
\text { indução da anestesia, utilizando grupo controle. A presença de } \\
\text { palhaços durante esse momento, em conjunto com os pais, foi } \\
\text { uma intervenção eficaz para controlar a ansiedade, apesar da } \\
\text { oposição da equipe pela interferência causada. }\end{array}$ \\
\hline $\begin{array}{l}\text { Vagnoli L et al., } \\
2010^{27}\end{array}$ & $\begin{array}{l}\text { Parental presence, } \\
\text { clowns or sedative } \\
\text { premedication to treat } \\
\text { preoperative anxiety in } \\
\text { children: what could } \\
\text { be the most promising } \\
\text { option? }\end{array}$ & $\begin{array}{l}\text { Investigou comparativamente a eficácia da palhaçoterapia, } \\
\text { do uso de midazolam ou da presença dos pais na redução da } \\
\text { ansiedade pré-operatória utilizando grupos controle. Conclui-se } \\
\text { que a intervenção clown é mais eficaz na redução da ansiedade } \\
\text { em crianças durante o período pré-operatório do que o } \\
\text { acompanhamento dos pais com ou sem o uso de midazolam. }\end{array}$ \\
\hline
\end{tabular}




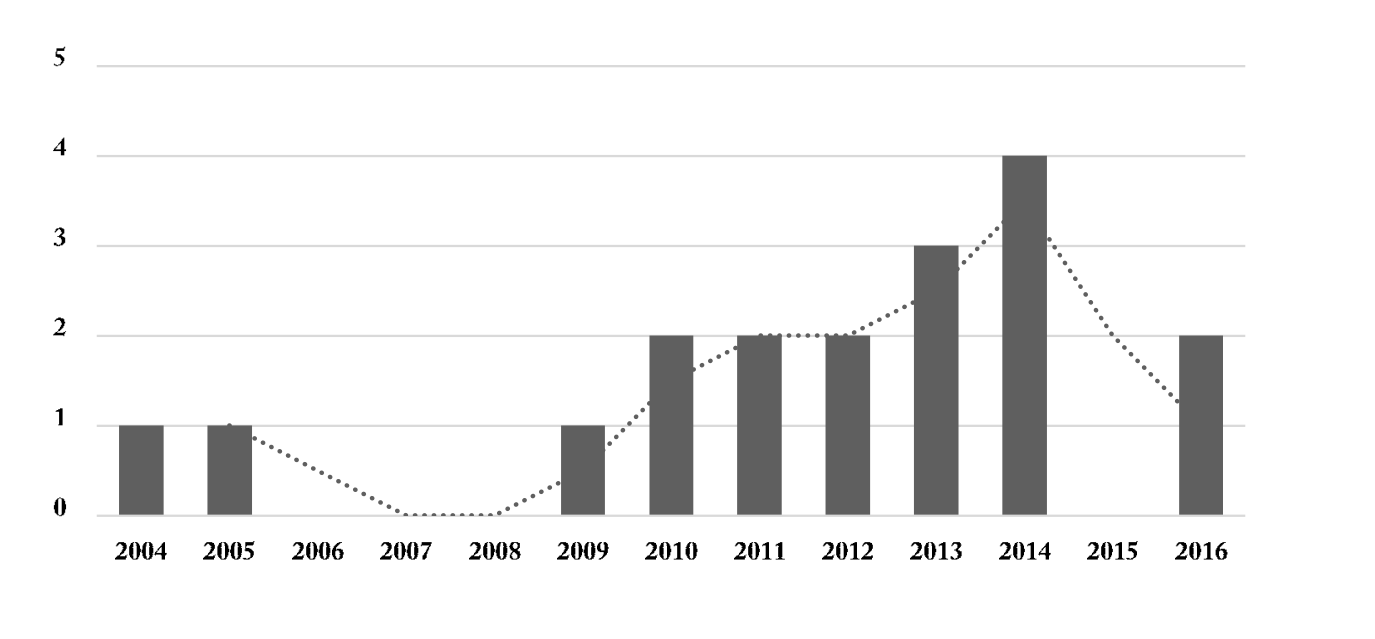

Gráfico 1. Número de trabalhos analisados por ano de publicação.

\section{Respostas fisiológicas, comportamentais e emocionais}

Kingsnorth et al. ${ }^{12}$ conduziram um estudo piloto em Toronto, no Canadá, avaliando repostas fisiológicas, comportamentais e emocionais em sete crianças hospitalizadas, com intervenções em quatro dias, alternando a palhaçoterapia com um programa de televisão escolhido pelo paciente. Ao final, uma entrevista com os pacientes, juntamente com dados fisiológicos coletados e observações de profissionais da equipe de enfermagem auxiliaram na discussão dos resultados. Concluíram que palhaçoterapia tem um impacto direto e positivo sobre as crianças hospitalizadas e ressaltaram a importância de abordagens alternativas na promoção do bem-estar dentro de ambientes de saúde ${ }^{12}$.

O estudo de Bertini et al. ${ }^{17}$ apontou para os benefícios da intervenção dos palhaços na evolução clínica de patologias respiratórias em quarenta e três crianças. Analisou parâmetros fisiológicos e de percepção de dor, concluindo que a presença de palhaços tem um possível efeito indutor de saúde e que o estímulo do bom humor pode ser visto como uma modalidade terapêutica fácil de usar, barata, natural e adaptável em diferentes ambientes e para diferentes necessidades terapêuticas. Os autores indicaram algumas limitações como a dificuldade de padronização do potencial das intervenções e a impossibilidade de generalização dos resultados, devido à baixa representatividade da amostra ${ }^{17}$.
Saliba et al. ${ }^{25}$ desenhou um estudo para correlacionar as atividades de palhaços com crianças hospitalizadas e o nível de cortisol, um importante biomarcador fisiológico do estresse. $\mathrm{O}$ cortisol salivar foi reduzido em todos os participantes que receberam a visita dos palhaços, demonstrando satisfação, indicando relativa atenuação dos efeitos da hospitalização e seu possível efeito colaborador no processo de recuperação da saúde ${ }^{25}$.

Os três estudos dessa categoria de análise são do tipo prospectivos, nos quais os pacientes foram expostos à situação em análise para então proceder a coleta de dados, com variáveis e instrumentos determinados previamente. Apesar de ter desenho definido e embasado, torna-se difícil o estabelecimento de relação de causalidade entre a exposição e o desfecho, dada impossibilidade de isolamento dos pacientes à outras exposições e a heterogeneidade característica da palhaçoterapia, pois os palhaços adaptam as intervenções de acordo com as necessidades apresentadas pelos pacientes naquele exato momento. Outra questão reside no fato destes estudos não abrangerem dimensões mais profundas ou que fujam aos resultados pré-estabelecidos para mensuração.

\section{Ansiedade pré-operatória}

A indução da anestesia, apontam Vagnoli et al. ${ }^{26}$, é um dos momentos mais estressantes para uma criança submetida a cirurgia sendo que altos níveis de ansiedade foram identificados como 
Quadro 3. Classificação dos artigos quanto ao país de origem, tipos de trabalho, população do estudo, métodos e categorias de análise.

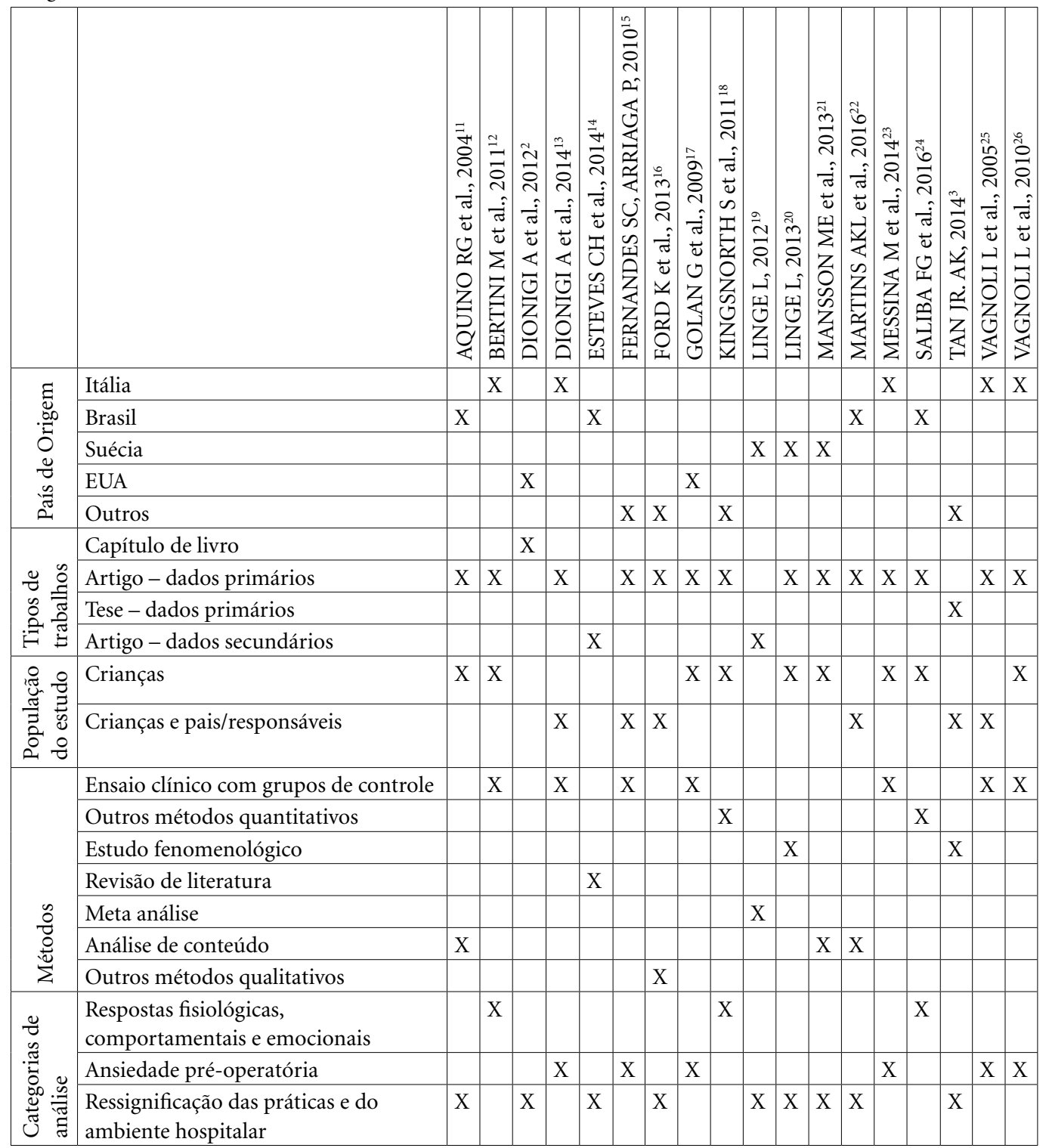

preditores de problemas pós-operatórios, que podem persistir por seis meses após o procedimento ${ }^{26}$.

Alguns trabalhos recentes utilizaram ensaios clínicos com grupos de controle para comparar os efeitos da palhaçoterapia e apontaram significativa diminuição nos níveis de ansiedade pré -operatória em crianças ${ }^{18,20,21,13,26,27}$. Dois deles afirmam que a palhaçoterapia é mais eficaz inclusive do que a administração de midazolam para reduzir essa ansiedade ${ }^{21,27}$.
Mansson et al. ${ }^{24}$ relatam que há crianças que não querem a presença dos palhaços durante procedimentos médicos e justificam seu achado citando o estudo realizado por Vagnoli et al. ${ }^{26}$. $\mathrm{Na}$ verdade, o último relata a resistência da equipe médica em relação a inclusão dos palhaços durante os procedimentos médicos e não dos pacientes, resultado semelhante ao de Messina et al. ${ }^{13}$. Já Fernandes e Arriaga ${ }^{20}$ relatam que os profissionais são favoráveis a intervenção dos palhaços. 


\section{Ressignificação das práticas e do ambiente hospitalar}

Sabe-se que internação hospitalar causa diversas mudanças na rotina habitual dos indivíduos e pode gerar sentimentos desfavoráveis ao restabelecimento do equilíbrio e a recuperação da saúde, além de exigirem elevada capacidade de $\operatorname{adaptação~}^{14}$.

Sofrimento, dor e pesar fazem parte da realidade hospitalar, tornando árdua a tarefa de incluir alegria neste ambiente. Enquanto a prática hospitalar não incorporar atitudes que visem o cuidado contínuo para além do físico, este espaço está fadado a ser um foco de doenças. E um dos objetivos da palhaçoterapia é eliminar esta lacuna, valorizado a individualidade e intersubjetividade particulares de cada caso $^{14}$.

A influência do humor na saúde tem sido positivamente reconhecida, relacionando-se com a redução da dor, com efeitos cardiovasculares e na imunidade, diminuição do estresse e aumento das habilidades sociais ${ }^{3}$.

Inicialmente, o paradoxo gerado pela inserção do palhaço no hospital pode causar certo estranhamento, já que neste local as condutas são guiadas pela seriedade, assertividade, precisão e cientificidade. Onde o toque é feito com luvas, o que, apesar de necessário, por vezes pode nos afastar do humano. E o palhaço nos traz a possibilidade do erro, de um ser atrapalhado, que por caminhos improvisados evidencia constantemente nossa fragilidade ${ }^{28}$.

O filósofo Henri Bergson ${ }^{29}$ traz uma reflexão sobre o cômico, expondo que o mesmo é propriamente humano, já que é feito por humanos e a respeito de humanos. Nenhum outro animal ri e uma paisagem ou objeto não se torna risível, a não ser que encontremos nisto certa expressão humana. Expressão que, a partir do desajeitamento, do involuntário, da mudança brusca de atitude, provoca o riso. $\mathrm{O}$ termo palhaço, originário da língua celta, designa um homem do campo visto pelas pessoas da cidade como desajeitado e engraçado ${ }^{28}$. Cometer erros faz parte da essência da palhaçaria ${ }^{15}$. Erro que não tem culpados e não enfurece ninguém. Observar a frustração do palhaço possivelmente facilita a aceitação da criança de sua própria condição, aumentando sua autoestima ${ }^{15}$.

A transformação do ambiente hospitalar se dá pela subversão da ordem normal das coisas ${ }^{15}$. A sátira realizada pelo "doutor" palhaço aos procedimentos e instrumentos hospitalares, assim como aos profissionais de saúde, são estratégias que proporcionam uma forma alternativa de enxergar a realidade. A especialidade médica passa a ser a besteira e o médico transforma-se em "besteirologista", o diagnóstico pode ser de "samba no pé" e os medicamentos passam a chamar-se “pílulas de bom humor" ou "pó de ficar bom”. Os uniformes brancos ganham um colorido, o silêncio dá lugar ao riso, às histórias e à música. $\mathrm{E}$ o medo do desconhecido é aliviado pelo inusitado.

Essa sátira não altera os fatos, mas sim seu significado, proporcionando ao paciente uma experiência menos desagradável, favorecendo o entendimento da realidade com a proteção da fantasia ${ }^{22}$. Promove a transformação do ambiente hospitalar em algo divertido, onde se pode rir e viver, mesmo dentro de suas limitaçõos ${ }^{23}$. Desmistifica procedimentos hospitalares e empodera os pacientes no domínio do corpo e na busca de sua saúde.

A naturalidade e alegria com que os palhaços lidam com a vida e a morte, denota que uma não é o oposto da outra ${ }^{28}$ e que, mesmo com a aproximação de ambas durante a internação hospitalar, isso faz com que a luta contra a morte passa a fazer parte da vida. Aquino et al. ${ }^{16}$ concluíram, que as crianças se sentem mais fortes, alegres, autônomas e que o palhaço pode diminuir as dores pela brincadeira e distração propostas. Masetti ${ }^{28}$ aponta que palhaços profissionais conseguem estabelecer relações de qualidade dentro dos hospitais, o que faz com que as crianças fiquem mais ativas, comunicativas, alimentem-se melhor, colaborem mais com o tratamento, além das evidências clínicas de melhora.

Diversos estudos demonstraram que a interação com os palhaços pode trazer benefícios tanto para trabalhadores de hospital quanto para pacientes, auxiliando na ressignificação deste ambiente $e^{3,19,15,23,14}$ outrossim visto como inóspito, estéril, silencioso e triste. Os palhaços alteram a atmosfera do hospital de modo geral ${ }^{15}$, normalmente gerando efeitos nos profissionais e em todos aqueles que encontram durante suas atuações.

A interação com os palhaços promove o contato com o tempo presente, já que o palhaço opera em um sistema de crenças e valores diferentes, desafiando a ordem social, sabotando a lógica e a racionalidade, sem linearidade entre os fatos ${ }^{28}$. Sua atitude é sempre imprevisível propondo soluções inesperadas para situações reais. Isso causa curiosidade e prende o expectador no aqui e agora, reconectando-o com o momento presente.

Bergson $^{29}$ relata que o maior inimigo do riso é a emoção. Portanto é necessária uma abstração momentânea das emoções presentes para que se 
possa rir. Essas sensações e vivências ficam registradas na memória, expandindo a percepção da realidade vivida, recontextualizando-a e ampliando cada vez mais seus próprios limites ${ }^{19,28}$.

Linge $^{23}$ aponta que a "área mágica e segura” criada pelo encontro com os palhaços se dá porque, quando brincam e testam suas possibilidades, as crianças reconhecem-se no estado em que estão naquele exato momento e não mais com foco na sua doença ou limitações. Isso aumenta a autoconfiança das crianças e as possibilidades de melhora. Empodera-o para lidar com a doença e incapacidade temporária, dando-o mais autonomia na busca pela sua própria saúde ${ }^{23}$.

O estabelecimento do vínculo e confiança com o palhaço e com a equipe de saúde favorece a comunicação, principal área afetada nessas circunstâncias, e a adaptação a um novo cenário durante esse processo. Enfermeiras relataram a diminuição do estresse e da ansiedade nos pacientes $^{28}$. Acelera-se a recuperação pós-operatória e a internação torna-se menos ameaçadora. Masettii ${ }^{28}$ aponta que a recuperação física do paciente está associada à liberação da energia despendida para lidar emocionalmente com a doença e a internação, já que ambos exigem alto grau de adaptação e elaboração. De forma análoga ao funcionamento dos sonhos, que nos auxiliam a lidar com conflitos e manter o equilíbrio físico e mental ${ }^{28}$.

Esteves et al. ${ }^{19}$ mostram que a humanização hospitalar já é um bem e uma necessidade reconhecida tanto ao nível teórico quanto na perspectiva dos usuários. Apesar da heterogeneidade das discussões sobre humanização do contexto hospitalar, sua importância no Brasil evidenciase pela criação do Programa Nacional de Humanização da Assistência Hospitalar(PNHAH), em 2001. A humanização deve ser vista em primeiro plano, independe do país ou regulamentação, buscando melhorar a qualidade de vida dos usuários e dos serviços prestados, promovendo o potencial terapêutico das relações positivas ${ }^{19}$.

As intervenções dos palhaços podem ser vistas como facilitadoras e promotoras diretas da humanização, já que transformam situações, trazem conforto e empoderamento, favorecem a comunicação e expressão. A interação dá voz à imaginação, desperta os sentidos, provoca emoções, amplia a visão individual, abrindo a possibilidade para um novo olhar e ampliando a percepção da realidade habitualmente construída ${ }^{19}$. Ademais, distrai a atenção e alivia a ansiedade de se permanecer isolado ${ }^{11}$; auxilia a lidar com o silêncio, com as emoções e com este novo am- biente, atendendo necessidades psicossociais dos pacientes $^{3}$.

\section{Considerações finais}

A palhaçoterapia é tema recente no campo de pesquisa científica e, apesar de ser realizada por diversos grupos em vários países, não há uniformidade em sua prática, tanto na formação dos profissionais que a executam quanto na forma como é realizada. O mesmo ocorre com sua utilização, efeitos esperados e público ao qual se direciona, o que dificulta a pesquisa e a produção de conhecimento científico e técnico sobre o assunto. Outro ponto que demanda investigações futuras refere-se as emoções decorrentes do humor nos pacientes, por vezes positivas ou negativas e, dada a heterogeneidade e espontaneidade característicos da palhaçoterapia, quais seriam os melhores métodos para avaliação dos efeitos causados.

Os resultados encontrados demonstraram majoritariamente benefícios nas diversas utilizações da palhaçoterapia em ambiente hospitalar, mesmo esta não sendo valorizada como prática promotora de saúde, de modo geral. Também indicaram que o uso da palhaçoterapia na redução da ansiedade pré-operatória parece seguir um desenho definido e trazer evidências consistentes. Outras pesquisas sobre alterações fisiológicas, comportamentais e emocionais em condições médicas específicas causadas por essa prática também vêm sendo investigadas em consonância com o modelo biomédico.

De modo geral, a palhaçoterapia tem boa aceitabilidade, mas há ainda resistência por parte dos profissionais de saúde. E, apesar da população estudada pelos autores dessa revisão serem, em sua maioria, crianças, esta prática também é realizada para pacientes adultos e idosos em diversas instituições hospitalares, o que demanda investigações futuras.

É possível afirmar que as alterações causadas no ambiente hospitalar pela inserção do palhaço parecem estar mais alinhadas com a filosofia desta prática, assim como ao conceito ampliado de saúde, desafiando o modelo biomédico atual que já não contempla integralmente as necessidades de saúde da população. Portanto espera-se que este estudo traga outros olhares para palhaçoterapia: uma intervenção considerada humanizadora e que integra diversas áreas do conhecimento concretizando-se no "fazer o bem, sem olhar a quem", algo raro atualmente. 


\section{Colaboradores}

SC Catapan participou de todas as etapas de elaboração do artigo. WF Oliveira orientou e participou de todas as etapas de construção do trabalho. TM Rotta participou da revisão crítica e aprovação da versão a ser publicada.

\section{Referências}

1. Oliveira WF. O núcleo de humanização, arte e saúde: uma experiência coletiva de produção social de saúde. Cadernos Brasileiros de Saúde Mental 2016; 8(18):198211.

2. Dionigi A, Flangini R, Gremigni P. Clowns in hospitals. In: Dionigi A, Flangini R, Gremigni P. Humor and Health Promotion. New York: Nova Science Publishers; 2012. p. 213-227.

3. Tan Junior AK. A Qualitative Phenomenographical Study of the Experience of Parents with Children in Clown Care Services [thesis]. Helsinki: Helsinki Metropolia University of Applied Sciences; 2014.

4. Adams H. Patch Adams: O amor é contagioso. Rio de Janeiro: Sextante; 1999.

5. Nogueira W. Doutores da Alegria: O lado invisível da vida. [S. 1.]: Mamo, 2006.

6. Masetti M. Por uma ética do encontro: a influência da atuação de palhaços profissionais na ação dos profissionais de saúde. Indagatio Didactica [periódico na Internet] 2013 [acessado 2016 Maio 17]; 5(2): [cerca de 13p.]. Disponível em: https://goo.gl/aOwOZj.

7. Moher D, Liberati A, Tetzlaff J, Altman DG, The PRISMA Group. Preferred Reporting Items for Systematic Reviews and Meta-Analyses: The PRISMA Statement. PLoS Med. [serial on the Internet] 2009 Jul [cited 2016 May 17]; 6(7): [about 4 p.]. Available from: https:// goo.gl/36MKoH.

8. Minayo MCS. Pesquisa Social: Teoria, método e criatividade. 29a ed. Petrópolis: Vozes; 2010.

9. Estomiolo Filho J. EndNote Web: guia de uso. 6a Atualização. São Paulo: Centro de Informação e Referência. São Paulo: Universidade de São Paulo; 2013.

10. Colletti G, Allevi F, Moneghini L, Palvarini M. Clown nose: a case of disfiguring nodular squamous cell carcinoma of the face. BMJ Case Rep [serial on the Internet] 2014 Jan 31. [cited 2016 May 17]: doi:10.1136/ bcr-2013-200471. [about 4 p.]. Available from: goo.gl/ lysGHR

11. Masetti M. Ética da Alegria no Contexto Hospitalar. $2^{\text {a }}$ ed. Rio de Janeiro: Folio Digital, Letra e Imagem; 2000.

12. Kingsnorth S, Blain S, Mckeever P. Physiological and emotional responses of disabled children to therapeutic clowns: a pilot study. Evid Based Complement Alternat Med [serial on the Internet] 2011 [cited 2016 May 17]; 732394: [about 10 p.] Available from: https://goo.gl/zBdwZd

13. Messina M, Molinaro F, Meucci D, Angotti R, Giuntini L, Cerchia E, Bulotta AL, Brandigi E. Preoperative distraction in children: hand-held videogames vs clown therapy. Pediatr Med Chir [serial on the Internet] 2014 Sep-Dec. [cited 2016 May 17]; 36(5-6): [about 3 p.]. Available from: https://goo.gl/2BVeKY

14. Martins AKL, Silva RG, Fernandes CM, Souza AMA, Vieira NFC. Repercussões da clownterapia no processo de hospitalização da criança. Rev pesqui cuid fundam [periódico na Internet] 2016 [acessado 2016 Maio 17]; 8(1): [cerca de 10 p.]. Disponível em: http:// goo.gl/7sfGww 
15. Ford K, Pratt HC, Tesch L, Johnson C. More than just clowns - Clown Doctor rounds and their impact for children, families and staff. Journal of Child Health Care. [serial on the Internet] 2013 [cited 2016 May 17]; 18(3): [about 11 p.]. Available from: https://goo. $\mathrm{gl} / \mathrm{Jff} 2 \mathrm{au}$

16. Aquino RG, Bortolucci RZ, Marta IER. Doutores da graça: a criança fala. Online braz. j. nurs. [periódico na Internet] 2004 [acessado 2016 Maio 17]; 3(2): [cerca de 8 p.]. Disponível em: http://goo.g1/55SVSP

17. Bertini M, Isola E, Paolone G, Curcio G. Clowns benefit children hospitalized for respiratory pathologies. Evid Based Complement Alternat Med [serial on the Internet] 2011 [cited 2016 May 17]; 2011: [about 9 p.]. Available from: goo.gl/pgr0pe

18. Dionigi A, Sangiorgi D, Flangini R. Clown intervention to reduce preoperative anxiety in children and parents: a randomized controlled trial. J Health Psychol [serial on the Internet] 2014 Mar [cited 2016 May 17]; 19(3): [about 12 p.] Available from: https://goo. $\mathrm{gl} / \mathrm{z} 7 \mathrm{e} 8 \mathrm{Kv}$

19. Esteves $\mathrm{CH}$, Antunes C, Caires S. Humanização em contexto pediátrico: o papel dos palhaços na melhoria do ambiente vivido pela criança hospitalizada. Interface (Botucatu) [serial on the Internet] 2014 [cited 2016 May 17]; 18(51): [about 12 p.]. Available from: http://goo.gl/xCcX2U

20. Fernandes SC, Arriaga P. The effects of clown intervention on worries and emotional responses in children undergoing surgery. J Health Psychol. [serial on the Internet] 2010 Apr [cited 2016 May 17]; 15(3): [about 10 p.]. Available from: https://goo.gl/9AmHNb

21. Golan G, Tighe P, Dobija N, Perel A, Keidan I. Clowns for the prevention of preoperative anxiety in children: a randomized controlled trial. Pediatric Anesthesia. [serial on the Internet] 2009 [cited 2016 May 17]; 19: [about 4 p.]. Available from: https://goo.gl/QGN4aI

22. Linge L. Magical attachment: Children in magical relations with hospital clowns. Int J Qual Stud Health Well-being. [serial on the Internet] 2012 [cited 2016 May 17]; 7:11862 [about 12 p.]. Available from:https:// goo.gl/O1y6fC

23. Linge L. Joyful and serious intentions in the work of hospital clowns: a meta-analysis based on a 7-year research project conducted in three parts. Int J Qual Stud Health Well-being. [serial on the Internet] 2013 [cited 2016 May 17]; 8: [about 8 p.]. Available from: https://goo.gl/f76GWa

24. Mansson ME, Elfving RN, Petersson C, Wahl J, Tunell S. Use of clowns to aid recovery in hospitalised children. Nurs Child Young People [serial on the Internet] 2013 Dec. [cited 2016 May 17]; 25(10): [about 5 p.]. Available from: https://goo.gl/oalGJh

25. Saliba FG, Adiwardana NS, Uehara EU, Silvestre RN, Leite VV, Faleiros FTV, Padovani FHP, Gobbi JIF Salivary Cortisol Levels: The Importance of Clown Doctors to Reduce Stress. Pediatr Rep [serial on the Internet] 2016 Mar 31 [cited 2016 May 17]; 8(6188): [about 3 p.]. Available from: https://goo.gl/36MKoH
26. Vagnoli L, Caprilli S, Robiglio A, Messeri A. Clown doctors as a treatment for preoperative anxiety in children: a randomized, prospective study. Pediatrics [serial on the Internet] 2005 Oct. [cited 2016 May 17]; 116(4): [about 5 p.] Available from: https://goo. $\mathrm{gl} / \mathrm{e} 2 \mathrm{tTEj}$

27. Vagnoli L, Caprilli S, Messeri A. Parental presence, clowns or sedative premedication to treat preoperative anxiety in children: what could be the most promising option? Paediatr Anaesth. [serial on the Internet] 2010 Oct. [cited 2016 May 17]; 20(10): [about 6 p.]. Available from: https://goo.gl/dkBkLX

28. Masetti M. Soluções de Palhaços: transformações na realidade hospitalar. $4^{a}$ ed. São Paulo: Aghena; 1998.

29. Bergson H. O riso: ensaio sobre a significação do cômico. $2^{\mathrm{a}}$ ed. Rio de Janeiro: Zahar; 1983.
Artigo apresentado em 17/01/2017

Aprovado em 29/01/2018

Versão final apresentada em 31/01/2018 
